\title{
Effect of Mode Stirrers in a Multimode Microwave- Heating Applicator with the Conveyor Belt
}

\author{
Harvey Kurniawan', Suresh Alapati', and Woo Seong Che ${ }^{1, t,}$ \\ 1 Department of Mechatronics Engineering, Kyungsung University, 309, Suyeong-ro, Nam-gu, Busan, South Korea, 608-736 \\ \# Corresponding Author / E-mail: wsche@ks.ac.kr, TEL: +82-51-663-4691, FAX: +82-51-626-4773
}

KEYWORDS: Microwave oven, Mode stirrers, Conveyor belt

\begin{abstract}
In this work, a numerical study is performed to know the effect of mode stirrers on the temperature distribution inside a microwave cavity with a conveyor belt system. A 3-dimensional simulation model is built and simulated using the COMSOL Multiphysics software. Since it is very difficult to simulate the mode stirrer's motion using COMSOL, eleven still angles of the mode stirrers are considered. The final temperature distribution result is obtained by taking the average of all eleven angles results. Also to get the final temperature of a heated material after it comes out of the cavity, the average of all temperature values in the direction of conveyor belt motion is taken as it is assumed that conveyor belt motion is much slower than the speed of the mode stirrers. The simulation results show that temperature distribution is more uniform for the case with mode stirrers compared to that obtained without mode stirrers. This is confirmed by using standard deviation equation.
\end{abstract}

Manuscript received: June 24, 2014 / Revised: September 15, 2014/ Accepted: September 16, 2014

\section{NOMENCLATURE}

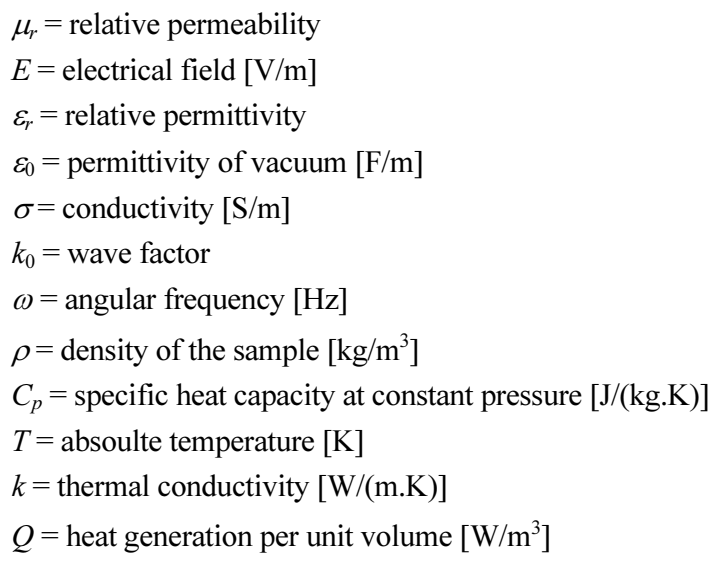

\section{Introduction}

Microwave-heating applicators have been widely used as a replacement for conventional oven for heating food in both household and industrial appliances. Microwave heating systems are rapidly spreading due to their high energy efficiency and faster heating time. ${ }^{1}$ Also, most of the microwave oven parts are recyclable. Since a microwave oven uses less energy compared to their traditional kitchen partners (gas and electric oven) and parts of it can easily be recycled, microwave heating system has become an eco-friendly (green) choice for cooking, baking, tempering, and pasteurizing food items. However, despite of its advantages, one of the major drawbacks of microwave heating is non-uniform heat distribution. Non-uniform heating of food may lead to decrease in the energy efficiency of microwave heating. Many ways have been proposed to achieve the uniform temperature distribution, including the use of mode stirrers in household microwave ovens, and conveyor belts in industrial purpose ovens.

Many studies have been conducted to increase the uniformity rate of microwave heating. In 2012, $\mathrm{Jun}^{2}$ did a study on how to increase uniformity by using multiple waveguides and proposed an equation called the uniformity equation to calculate the percentage of temperature uniformity. In 2013, Coman et al., studied the effect of placement of dielectric material inside a microwave cavity and determine the best placement using COMSOL Multiphysics and Minitab software. ${ }^{3}$

One of the methods to achieve the uniform distribution of heat inside a microwave cavity is through the use of mode stirrers. Most of the microwave ovens in household as well as in industrial appliances 
have been equipped with mode stirrers to increase the heat distribution uniformity. In 2004 and 2005, Plaza-González et al.,,5 did a study to know the effect of mode stirrers on the behavior of the electric field inside a microwave cavity. However they only performed simulations on a 2-dimensional cavity, which is different from the real scenario.

Another commonly used method to increase the uniformity of the temperature distribution is by using a conveyor belt. In 2010, Methlouthu et al., ${ }^{6}$ did a simulation of a real microwave cavity with conveyor belt using COMSOL Multiphysics in order to know the distribution of electric field inside the cavity. They only calculated the electric field and temperature distribution while assuming the static condition of the cavity as it is very difficult to evaluate the inside of a microwave cavity when conveyor belt is in motion.

In this work, we performed the numerical simulation on a 3dimensional microwave cavity using COMSOL Multiphysics. The main aim of this work is to know the effect of the mode stirrers and conveyor belt motion on the uniformity of the temperature distribution inside a microwave cavity. The remaining of this paper is arranged as follows. A brief description of mathematical model is given in Section 2. Section 3 discusses about the validation of COMSOL Multiphysics results with the experimental results. The simulation results are discussed in Section 4. Finally, the concluding remarks of the present work are given in Section 5 .

\section{Mathematical Model}

The heating process in microwave oven is a combination between the physics of electromagnetic and thermal fields.

The wave equation of the electromagnetic field is solved by using the following equation:

$$
\nabla \times \mu_{r}^{-1}-k_{0}^{2}\left(\varepsilon_{r}-j \sigma / \omega \varepsilon_{0}\right) E=0
$$

and the heat transfer equation is used to solve the thermal problem: ${ }^{7}$

$$
\rho C_{p} \frac{\partial T}{\partial t}+\nabla \cdot(-k \nabla T)=Q
$$

In order to obtain the temperature distribution inside the microwave cavity, the above equations are solved using COMSOL Multiphysics version 4.0.

\section{Validation of COMSOL Result}

In order to verify the simulation result given by COMSOL, a comparison between COMSOL result and experimental result is made. By using the 2-dimensional (2D) cavity size and material properties parameters that were defined in the reference, ${ }^{4}$ a $2 \mathrm{D}$ model is developed and simulated using COMSOL.

Fig. 1 shows the comparison of present simulation results, experimental results, and simulation results of Plaza-González et al.. ${ }^{4}$ It can be seen from Fig. 1 that the present simulation results are in better agreement with the experimental results than the previous simulation results. ${ }^{4}$ From this comparison we can say that our simulation

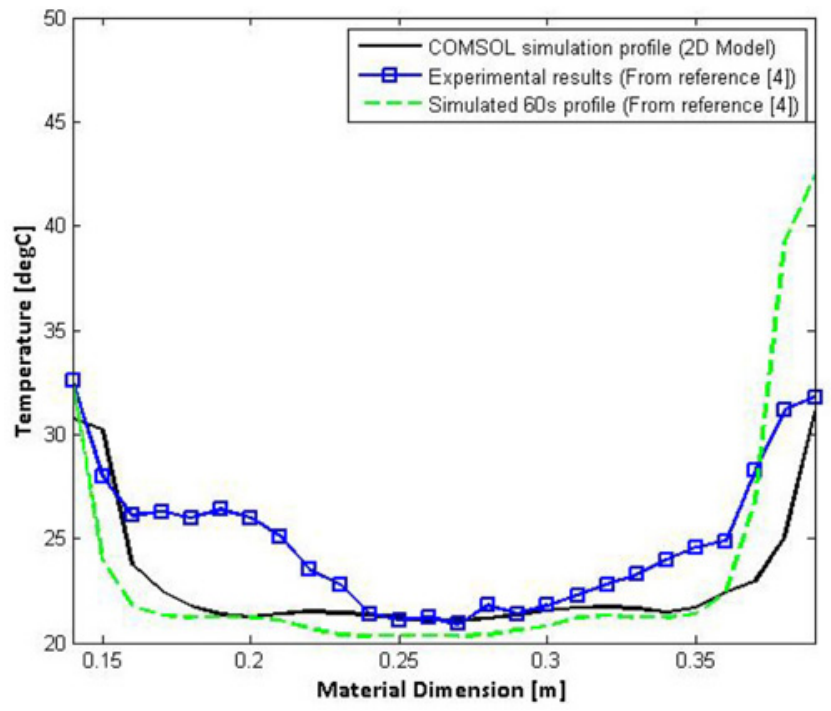

Fig. 1 Validation of COMSOL Multiphysics result with the previously published data ${ }^{4}$ (all simulations performed with 60 seconds heating time)

procedure of using COMSOL Multiphysics is correct. As we can see from Fig. 1 that the temperature is not uniform in the heated material as there are no mode stirrers inside the micro-wave cavity.

\section{Simulation Results}

\subsection{Simulation Method}

After the validation of 2-dimensional model, 3-dimensional models of microwave cavities with and without mode stirrers are developed using COMSOL in order to study the effect of mode stirrers on the temperature distribution inside the cavity. Fig. 2 and 3 show the microwave cavities without and with mode stirrers, respectively. The dimensions of both cavities are kept same. The length, width, and height of each cavity are taken as $0.593[\mathrm{~m}], 0.5[\mathrm{~m}]$, and $0.5[\mathrm{~m}]$, respectively. A glass plate is positioned (at 0 to $0.593[\mathrm{~m}]$ in the $\mathrm{x}$ direction, -0.25 to $0.25[\mathrm{~m}]$ in the $y$-direction, and 0.05 to $0.055[\mathrm{~m}]$ in the z-direction) on the bottom surface of the cavity and the heated sample (sawdust in this work) is mounted on the glass plate. The electromagnetic wave enters the cavity from the 2 WR-340 type waveguides located on top of the cavity which are excited with its dominant mode $\mathrm{TE}_{10}$. Two stirrers (each positioned at $-0.08[\mathrm{~m}]$ and at $0.08[\mathrm{~m}]$ in the $y$-direction, respectively) are attached to the roof of the cavity for the case of microwave cavity with mode stirrers. Steel is used to cover both the outer surface of cavity and the stirrer walls. The parameters (such as the sample's dielectric and thermal properties) used in the present simulation are listed in Table 1.

In real scenario, the mode stirrers will swing from left to right continuously when the material is moving through the conveyor belt. Because of the difficulty in simulating moving mode stirrers, we used the same method proposed by Plaza-González et al., who divided the movement of the mode stirrers into several static angles. This assumption was valid because they considered that the speed of the mode stirrers was much faster than the heating rate of the sample. In this work, since we also taken the conveyor belt motion into account, 


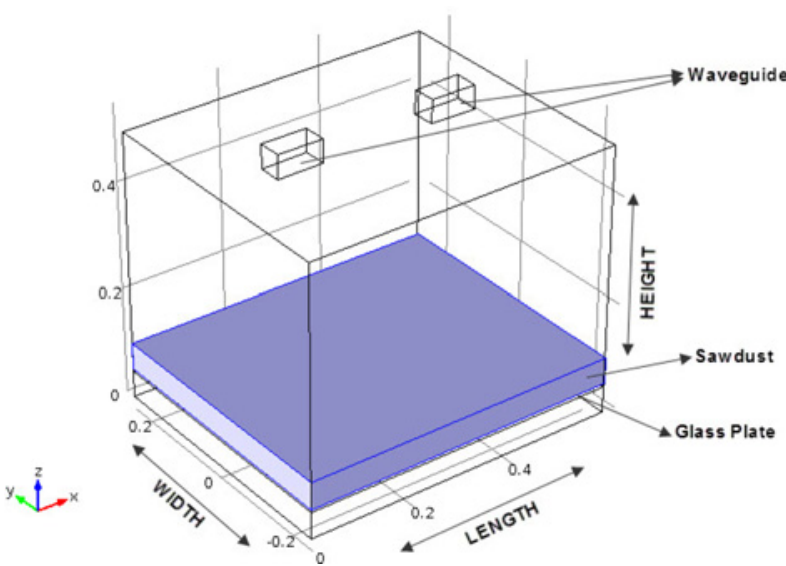

Fig. 2 Cavity without mode stirrers

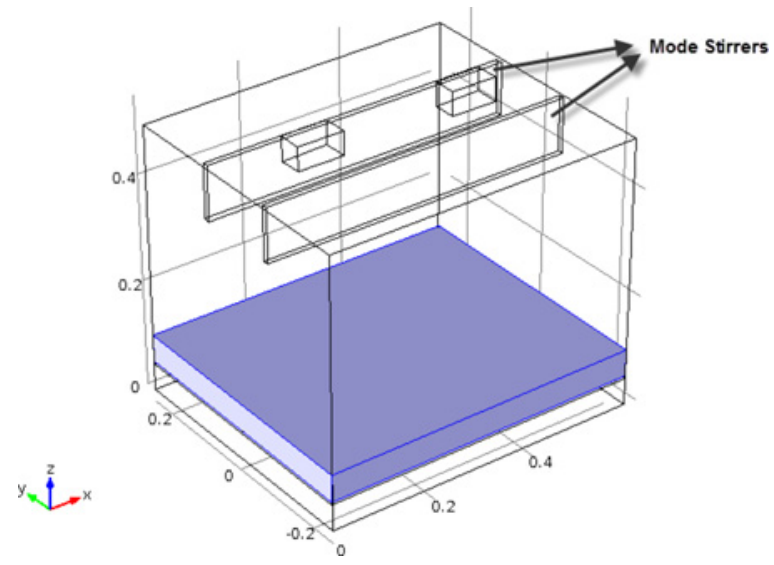

Fig. 3 Cavity with mode stirrers

Table 1 Simulation parameters of the sample

\begin{tabular}{cc}
\hline Moisture Content & $20[\%]$ \\
\hline Density & $200\left[\mathrm{~kg} / \mathrm{m}^{3}\right]$ \\
\hline Relative Permittivity & $1.6-0.21 \mathrm{j}$ \\
\hline Relative Permeability & 1 \\
\hline Heat Capacity & $1.4 \times 10^{3}\left[\mathrm{~J} /\left(\mathrm{kg} \cdot \mathrm{m}^{3}\right)\right]$ \\
\hline Thermal Conductivity & $0.08[\mathrm{~W} /(\mathrm{m} . \mathrm{K})]$ \\
\hline Penetration Depth & $0.117[\mathrm{~m}]$ \\
\hline Thickness & $0.05[\mathrm{~m}]$ \\
\hline
\end{tabular}

the speed of the mode stirrers is considered to be much faster than the speed of the conveyor belt. Also because of the difficulty in simulating the motion of the conveyor belt, in this work, a simple summation method is proposed to imitate the motion of the conveyor belt (will be described in detail in the next section).

In order to save computational time, at first, a simulation study is performed to know the relation between heating time and temperature distribution in the sample. The cavity without mode stirrers is used as the simulation model, with heating time of 60 seconds. It should be noted that all the results reported in this work are taken at the middle layer of the sawdust. Fig. 4 shows the contours of temperature and electric field distribution in the sample at different times. It is seen that the distribution of heat spots in the heated sample does not change with time as the electric field distribution does not vary with time. Therefore, the temperature of the sample varies linearly with time as shown in Fig. 5. So, in this
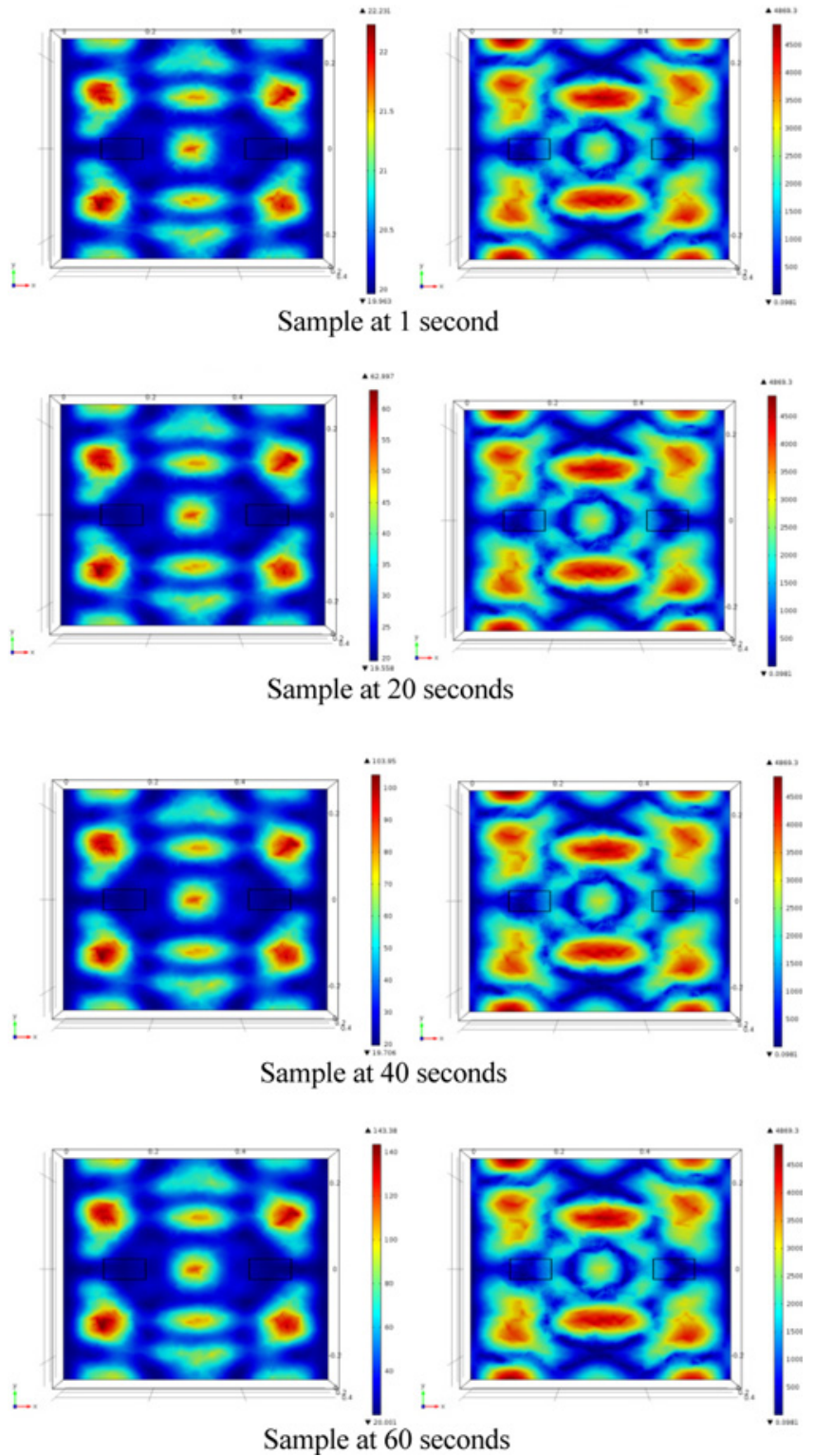

Fig. 4 Temperature (left) and electric field (right) distribution in the heated sample at $1,20,40$, and 60 seconds

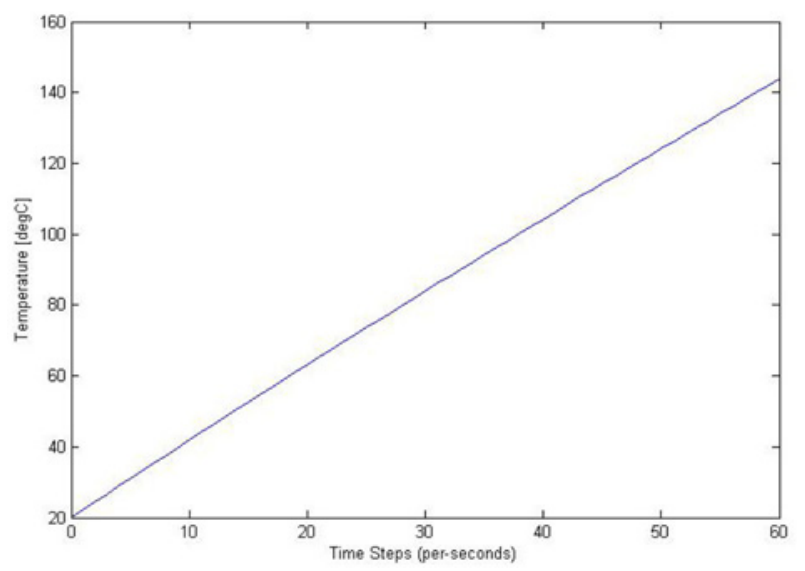

Fig. 5 Relation between time and temperature

work, we considered the heating time of only 1 second in order to minimize the total calculation time. 

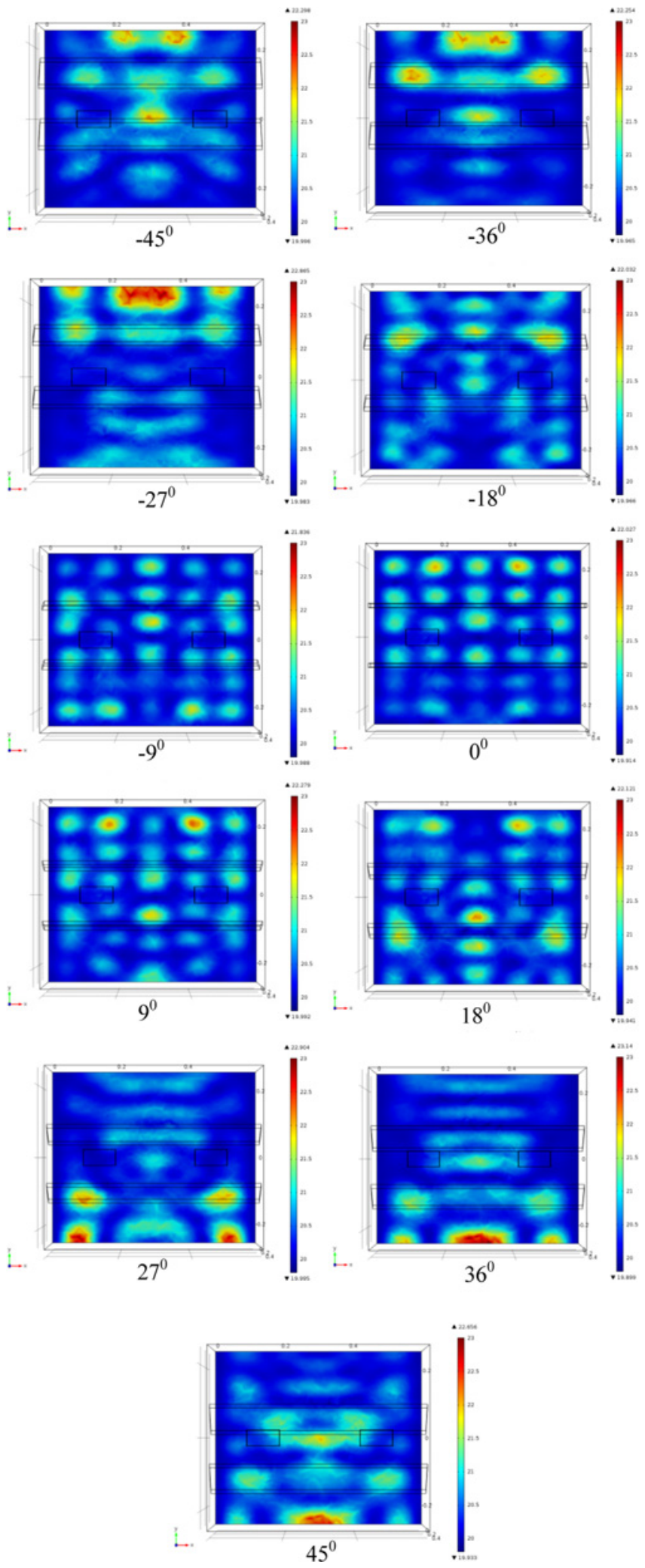

Fig. 6 Temperature distribution results of eleven angles

\subsection{Simulation of Cavity with Mode Stirrers}

As mentioned before, simulation of the cavity with mode stirrers is performed by dividing the moving stirrers into several static angles ranging from $-45^{\circ}$ to $45^{\circ}$ with 9 degrees increment. Fig. 6 shows the temperature distribution results of each angle. The temperature results of all the above angles are summed and averaged to get the final

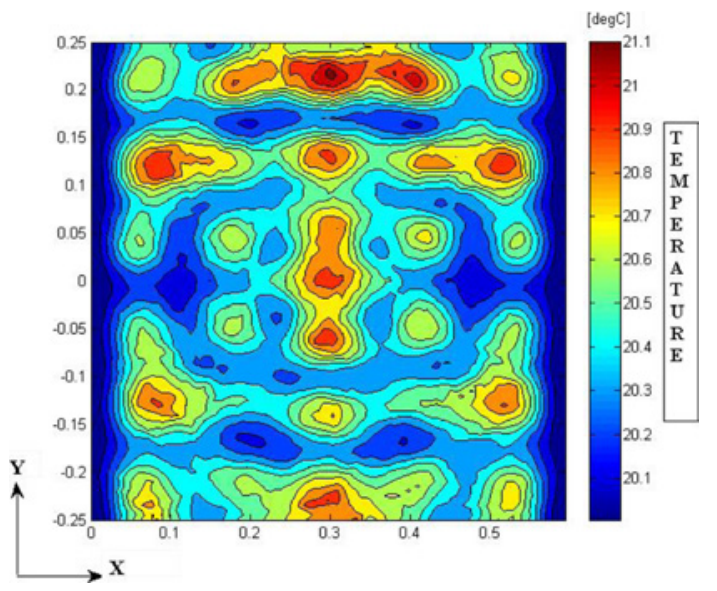

Fig. 7 Temperature distribution inside the cavity for the case of moving mode stirrers

temperature distribution result (Fig. 7) of the moving mode stirrers.

From Fig. 7, it is seen that the temperature distribution result of microwave cavity with moving mode stirrers is more uniform (more heat spots) compared to that without mode stirrers (Fig. 4). This means that mode stirrers provide better uniformity of the heat distribution inside the heated sample.

The non-uniformity of temperature distribution inside the cavity without mode stirrers can greatly affect the sample's heating rate. If the sample is potato chips placed on the top of a conveyor belt, which is moving either in the $\mathrm{x}$-direction or in the $\mathrm{y}$-direction, some chips may not cook at all because of the low temperature area in some locations or some chips may burn because of the very high temperature area. On the other hand, for the case of the cavity with mode stirrers, since heat distribution inside the sample spreads quite evenly, the cooking process is more uniform.

\subsection{Cavity with Conveyor Belt Motion}

As already mentioned, to get the final temperature distribution of the cavity with conveyor belt, we used a results summation method that imitate the conveyor belt motion as it is very difficult to simulate the conveyor belt motion using COMSOL. The idea of this method is to divide the 2-dimensional result of temperature distribution into several mesh points (say $101 \times 101$ in $\mathrm{x}$ and $\mathrm{y}$ directions) and take the average of the result in the direction of conveyor belt motion. Further explanation of this method is given as follows (see Fig. 8). Each of the mesh points shown in Fig. 8 contains temperature data of that particular point. Then assume the mesh points on one edge of the cavity where the sample to be heated enters the cavity on a conveyor belt. The rest of the mesh points pointing towards the opposite side the cavity are the points that the sample will go through before it gets to the exit of the cavity. To get the final temperature of the product, all the temperature data of the mesh points in the direction of conveyor belt motion are summed up and averaged.

As shown in Fig. 8, the entering sample is marked with the hollow rectangle and the exit of the cavity is marked with the "FINAL PRODUCT" sign. For example for Fig. 7, if temperature result data is divided into $[101 \times 101]$ meshes, the entering sample will contain [101×1] mesh points. Then after the summation with regards to conveyor belt motion direction, the final product temperature also 


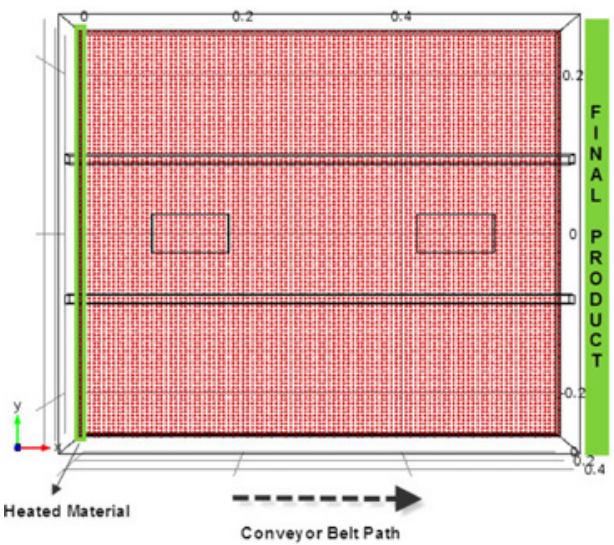

(a)

$x$-axis conveyor belt motion

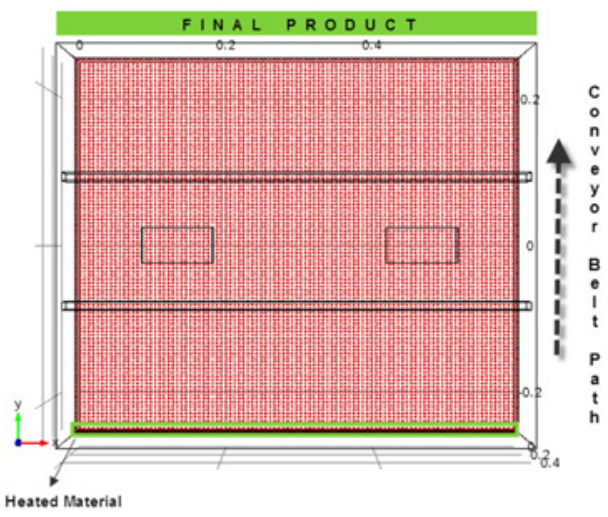

(b)

y-axis conveyor belt motion

Fig. 8 Conveyor belt movement in x-direction (left) and y-direction (right)
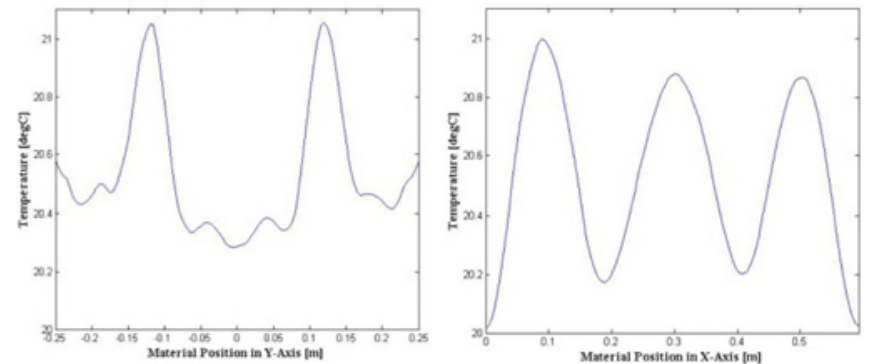

Fig. 9 Temperature distribution of final product without mode stirrers when the conveyor belt is moving in $\mathrm{x}$ - (left) and $\mathrm{y}$ - (right) direction
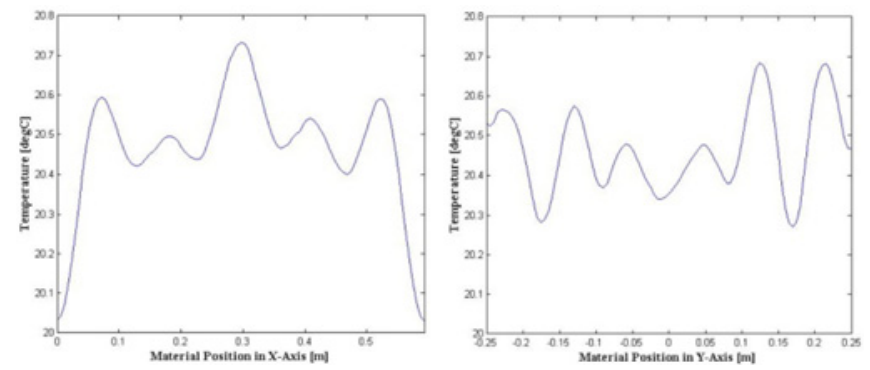

Fig. 10 Temperature distribution of final product with mode stirrers when the conveyor belt is moving in $\mathrm{x}$ - (left) and $\mathrm{y}$ - (right) direction

Table 2 Standard deviation result of all cases

\begin{tabular}{cc}
\hline Case & Standard Deviation Result \\
\hline $\begin{array}{c}\text { Without mode stirrers } \\
\text { (conveyor belt x-axis motion) }\end{array}$ & 0.218754 \\
\hline $\begin{array}{c}\text { With mode stirrers } \\
\text { (conveyor belt x-axis motion) }\end{array}$ & 0.106829 \\
\hline $\begin{array}{c}\text { Without mode stirrers } \\
\text { (conveyor belt y-axis motion) }\end{array}$ & 0.282844 \\
\hline $\begin{array}{c}\text { With mode stirrers } \\
\text { (conveyor belt y-axis motion) }\end{array}$ & 0.155617 \\
\hline
\end{tabular}

contains $[101 \times 1]$ mesh points of temperature data, which is to be plotted to get the temperature profile.

Fig. 9 and 10 show the temperature distribution inside the microwave cavity without (up) and with (down) mode stirrers when the conveyor belt is moving in the $\mathrm{x}$ - and $\mathrm{y}$-directions. Even though the conveyor belt motion considered into account, the temperature distribution of both the cases (cavity without and with mode stirrers) follows similar trend of that without conveyor belt motion. Comparing the results in Fig. 9 and 10, we can say that the heat distribution inside the cavity with mode stirrers is more uniform compared to that without mode stirrers.

Since the standard deviation (SD) is an indication of uniformity of the data, we calculated the SD of the temperature distribution results of all the cases. SD results of all the cases are displayed in Table 2, which shows that the cavity with mode stirrers has less SD comparing to the cavity without mode stirrers. This means that the temperature inside the cavity with mode stirrers is more uniformly distributed compared to that without mode stirrers.

\section{Conclusions}

In this work, the effect of mode stirrers on the temperature distribution inside a microwave cavity with a conveyor belt system was studied using COMSOL Multiphysics software. The main motivations of this work are to improve the quality of the multimode microwave-heating used in the industrial applications as such microwave applicators usually equipped with a conveyor belt system to improve the productivity, and to help developing a better heating system that minimizes energy intake.

To validate the solution given by COMSOL, the simulation results of the temperature distribution inside the microwave cavity were first compared with the existing experimental result. ${ }^{4}$ It was found that there is a qualitative agreement between the two results i.e. the temperature profile results of simulation and experiments follow similar trend even though there is a slight deviation between the results. It is thought that this deviation is due to the simulation conditions that are different from the experimental conditions. After this verification, simulations were performed on a multimode microwave cavity with a conveyor belt system.

At first, simulations were performed inside the microwave cavity with and without mode stirrers. It was observed that the positions of heat spots in the heated material remain same while temperature increases linearly with time. This is because electric field distribution doesn't change with time. So, in order to minimize computational time, simulations were performed by considering heating time of only 1 second. For the case of microwave cavity without mode stirrers, even though heat spots show 
quite symmetrical pattern, the temperature distribution is not uniform (temperature is very low at the middle of the cavity). More uniform distribution of temperature is obtained when the simulations were performed with mode stirrers. Later, the motion of the conveyor belt is considered to know its effect on the temperature distribution.

It is assumed that the conveyor belt motion is slower than the oscillating speed of mode stirrers. So, all the temperature values in the direction of conveyor belt motion were summed and averaged in order to get the final result of temperature distribution at the exit of microwave cavity. Simulation results show that the combination of conveyor belt with mode stirrers indeed increases the sample's temperature uniformity distribution. Also, a standard deviation equation was used to know the deviation of the temperature from the average (uniform temperature). It was found that with mode stirrers, the deviation in the temperature profile of the heated material reduced by around $50 \%$ which is quite a significant result.

\section{ACKNOWLEDGEMENT}

This research was supported by Kyungsung University Research grants in 2014.

\section{REFERENCES}

1. McConnell, D. R., "Energy Consumption: A Comparison between the Microwave Oven and the Conventional Electric Range," The Journal of Microwave Power, Vol. 9, No. 4, pp. 341-347, 1974.

2. Jun, M., "The Study on Microwave Drying System for Food with Multiple Waveguide," M.Sc. Thesis, Department of Mechatronics Engineering, Kyungsung University, 2012.

3. Coman, S., Leuca, T., Coman, O., and Laza, M., "Statistical Optimization of Dielectric Material's Placement inside a Microwave Applicator using Response Surface Method," Proc. of the International Symposium on Advanced Topics in Electrical Engineering, pp. 1-6, 2013.

4. Plaza-González, P., Monzó-Cabrera, J., Catalá-Civera, J. M., and Sánchez-Hernández, D., "New Approach for the Prediction of the Electric Field Distribution in Multimode Microwave-Heating Applicators with Mode Stirrers," Proc. of the IEEE Transactions on Magnetics, Vol. 40, No. 3, pp. 1672-1678, 2004.

5. Plaza-González, P., Monzó-Cabrera, J., Catalá-Civera, J. M., and Sánchez-Hernández, D., "Effect of Mode-Stirrer Configurations on Dielectric Heating Performance in Multimode Microwave Applicators," Proc. of the IEEE Transactions on Microwave Theory and Techniques, Vol. 53, No. 5, pp. 1699-1706, 2005.

6. Methlouthi, A., Rouaud, O., and Boillereaux, L., "Microwave Applicator with Conveyor Belt System," Proc. of the COMSOL in Paris, 2010.

7. COMSOL Inc., "COMSOL Multiphysics User Guide,” 2011. 